\title{
Derivation and validation of the CANP scoring model for predicting the neurological outcome in post-cardiac arrest patients
}

Gannan Wang, MD, Zhongman Zhang, MD, Xiaoquan Xu, MD, Qingsong Sun, MD, Haichen Yang, MD, Jinsong Zhang, MD, PhD.

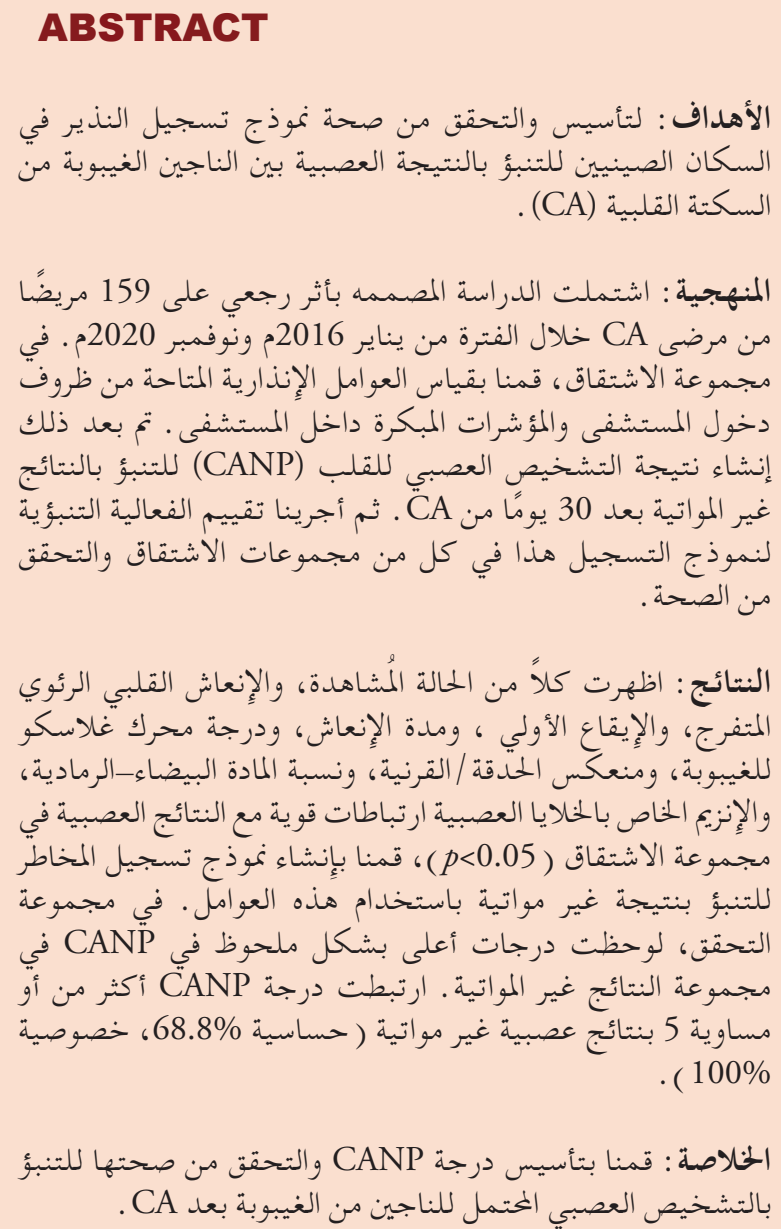

Objectives: To establish and validate a prognostic scoring model in a Chinese population to predict the neurological outcome among comatose survivors of cardiac arrest (CA).

Methods: 159 CA patients between January 2016 and November 2020 were recruited in this retrospective study. In the derivation cohort, prognostic factors available from arrest circumstances and early inhospital indicators were measured. The Cardiac
Arrest Neurological Prognosis (CANP) score was then constructed to predict unfavorable outcomes at 30 days after CA. The assessment of predictive effectiveness of this scoring model was conducted in both derivation and validation cohorts.

Results: Witnessed status, bystander cardiopulmonary resuscitation, initial rhythm, duration of resuscitation, Glasgow Coma Scale motor score, pupillary/ corneal reflex, gray-white matter ratio and neuronspecific enolase exhibit strong correlations with the neurological outcomes in the derivation cohort (all $p<0.05$ ), and a risk scoring model for the prediction of an unfavorable outcome was created using these factors. In the validation cohort, significantly higher CANP scores were noted in the unfavorable outcome group. A CANP score $\geq 5$ was associated with unfavorable neurological outcomes (sensitivity $68.8 \%$, specificity $100 \%)$.

Conclusion: The CANP score was established and validated for predicting the possible neurological prognosis in comatose post-CA survivors.

Neurosciences 2021; Vol. 26 (4): 372-378 doi: 10.17712/nsj.2021.4.20210056

From the Department of Emergency (Wang, Z Zhang, J Zhang), Department of Radiology $(X u)$, the First Affiliated Hospital of Nanjing Medical University, Nanjing, Jiangsu 210029, China; Department of Emergency (Sun), the Affliated Huaian No.1 People's Hospital of Nanjing Medical University, Huaian, Jiangsu 223300, China; Department of Emergency (Yang), the Affliated Huaian Hospital of Xuzhou Medical University, Huai'an, Jiangsu 223002, China

Received 22nd June 2021. Accepted 1st September2021.

Address correspondence and reprint request to: Dr. Jinsong Zhang, Department of Emergency, the First Affliated Hospital of Nanjing Medical University, Nanjing, Jiangsu 210029, China. E-mail: wgn0819jsph@vip.163.com

ORCID ID: https://orcid.org/0000-0002-5327-2750 
$\mathrm{C}$ ardiac arrest (CA) is among the main causes of mortality in modern medicine. Continuous improvements in managing CA patients, such as highquality cardiopulmonary resuscitation (CPR) with early defibrillation, have made it possible for more survivors to achieve return of spontaneous circulation (ROSC); however, a substantial number of even optimally managed victims experience devastating neurological outcomes, despite "successful" resuscitation. ${ }^{1}$ Early prognostication might aid in allocating treatment, and for providing sufficient information to their relatives. ${ }^{2}$ For comatose patients after ROSC, the current guidelines recommend a multimodal neurological prognostication algorithm on the basis of clinical examination at 72 hours or later after CA and several additional approaches, including neuroelectrophysiology, neuroimaging, and serum biomarkers. ${ }^{3}$ However, there is not a single clinical protocol, examination or test perfect for the determination of neurological prognosis given the variability in brain injury patterns noted in CA patients. ${ }^{4}$

Recent studies have expanded the prediction models and scoring systems to support early prediction as well as risk stratification in patients with ROSC after CA. ${ }^{5-9}$ These approaches share satisfactory performance but they vary widely in terms of their components and cause difficulties in obtaining reproducibility and external validation. ${ }^{10}$ These scores have improved the evaluation of the severity in CA patients in spite of the heterogeneity. However, these scoring models fail to take some known prognostic factors into account and have not been validated in different populations. Therefore, this study was to establish a prognostic scoring model in a Chinese population to predict the neurological outcomes among comatose survivors of CA. In the analysis, the first step was to determine the association between prognostic factors available from arrest circumstances and early in-hospital indicators and unfavorable outcomes at 30 days in a derivation cohort. The second step was to construct a clinically useful scoring system for the prediction of an unfavorable outcome and to perform an external validation.

Disclosure. This study was supported by Ruiyi special fund for emergency medical research (R2019019) and Postgraduate Research \& Practice Innovation Program of Jiangsu Province (SJCX20_0481). Authors have no conflict of interests, and the work was not supported or funded by any drug company.
Methods. This was a multicenter retrospective observational study recruiting patients with CA in the period from January 2016 to November 2020. Patients with persistent coma after ROSC were included, defined as a Glasgow Coma Scale (GCS) score $\leq 8$ for greater than $20 \mathrm{~min}$. Within $72 \mathrm{~h}$ after ROSC, brain $\mathrm{CT}$ was conducted for all eligible patients. Those under 18 years of age with terminal malignancy and baseline neurological impairment were excluded. In addition, patients with CT images that showed abnormal parenchyma or were technically insufficient to determine cerebral density or were unable to be assessed were also excluded. ${ }^{11}$ The flow chart of case enrollment is presented in Figure 1. 159 patients were enrolled in this study. The derivation cohort consisted of 80 patients receiving treatment at the First Affiliated Hospital of Nanjing Medical University (Nanjing, China). To evaluate general applicability, external validation in another cohort, including 79 patients treated in two other hospitals, namely, Huai'an First People's Hospital and Huai'an Second People's Hospital (Huai'an, China), was performed. Our study was granted approval by the Ethics Committee of each hospital and was conducted according to the Declaration of Helsinki.

Data collection. Data, including age, gender, situation surrounding CA, witnessed status, bystander $\mathrm{CPR}$, initial rhythm, and resuscitation duration (from CPR to ROSC) were systematically recorded in accordance with the Utstein style. ${ }^{11}$ Furthermore, all the clinical pictures and available prognostic indicators such as clinical exams, gray-white matter ratio (GWR) by CT scan, and serum neuron-specific enolase (NSE) were assessed. Historic prognostic predictors were combined with additional clinical information, including GWR and NSE, and a multimodal approach to prognostication was applied. The historic predictors of unfavorable neurological outcome were taken into consideration if any of the following existed: extensor posturing to external stimuli or absent motor response [GCS-motor response (GCS-M) $\leq 2$ ], no pupillary reflexes, or no corneal reflexes. GCS-M and pupillary/ corneal reflexes should be determined at least $72 \mathrm{~h}$ after CA excluding the effects of neuromuscular blocking drugs or sedatives.

The GWR was calculated by the methodology described in our previous study. ${ }^{12}$ All CT examinations (with 5-mm slice thickness) were performed within $72 \mathrm{~h}$ after resuscitation. On 3 axial slices (basal ganglia, centrum semiovale, and high convexity), two experienced investigators independently located the regions of interests (ROIs), in which attenuation values of the gray and white matter were measured. Both investigators were blinded to clinical outcomes, patient 
Table 1 - Baseline characteristics of the derivation and validation cohorts.

\begin{tabular}{|c|c|c|}
\hline Variable & $\begin{array}{l}\text { Derivation cohort } \\
\quad(\mathrm{n}=80)\end{array}$ & $\begin{array}{l}\text { Validation cohort } \\
\quad(\mathrm{n}=79)\end{array}$ \\
\hline Age (years), mean $\pm S D$ & $50.23 \pm 18.89$ & $54.57 \pm 14.40$ \\
\hline Male, n (\%) & $54(67.5)$ & $45(57.0)$ \\
\hline Witnessed, n (\%) & $60(75.0)$ & $71(89.9)$ \\
\hline Bystander CPR, n (\%) & $55(68.8)$ & $33(41.8)$ \\
\hline Out-of-hospital CA, n (\%) & $47(58.8)$ & $49(62.0)$ \\
\hline \multicolumn{3}{|l|}{ Comorbidities, $n(\%)$} \\
\hline Coronary artery disease & $9(11.3)$ & $10(12.7)$ \\
\hline Hypertension & $31(38.8)$ & $35(44.3)$ \\
\hline Diabetes & $14(17.5)$ & $17(21.5)$ \\
\hline Stroke & $9(11.3)$ & $8(10.1)$ \\
\hline Initial rhythm, shockable, n (\%) & $29(36.3)$ & $21(26.6)$ \\
\hline Cardiac etiology, n (\%) & $41(51.2)$ & $24(30.4)$ \\
\hline Duration of resuscitation (min), median (IQR) & $31.00(17.75-50.00)$ & $20.00(12.00-40.00)$ \\
\hline Length of stay in hospital (d), median (IQR) & $11.00(4.00-22.75)$ & $12.00(6.00-19.00)$ \\
\hline GCS-M $\leq 2, \mathrm{n}(\%)$ & $61(76.3)$ & $54(68.4)$ \\
\hline Pupillary/corneal reflex, n (\%) & $52(65.0)$ & $39(49.4)$ \\
\hline \multicolumn{3}{|l|}{$G W R$, median $(I Q R)$} \\
\hline Basal ganglia & $1.18(1.11-1.29)$ & $1.18(1.10-1.28)$ \\
\hline Average & $1.24(1.12-1.35)$ & $1.22(1.16-1.33)$ \\
\hline NSE (ng/ml), median (IQR) & $83.51(36.28-243.90)$ & $86.87(36.30-300.00)$ \\
\hline \multicolumn{3}{|l|}{ CPC at 30 days, $n(\%)$} \\
\hline CPC 3-5 & $58(72.5)$ & $55(69.6)$ \\
\hline CPC 1-2 & $22(27.5)$ & $24(30.4)$ \\
\hline
\end{tabular}

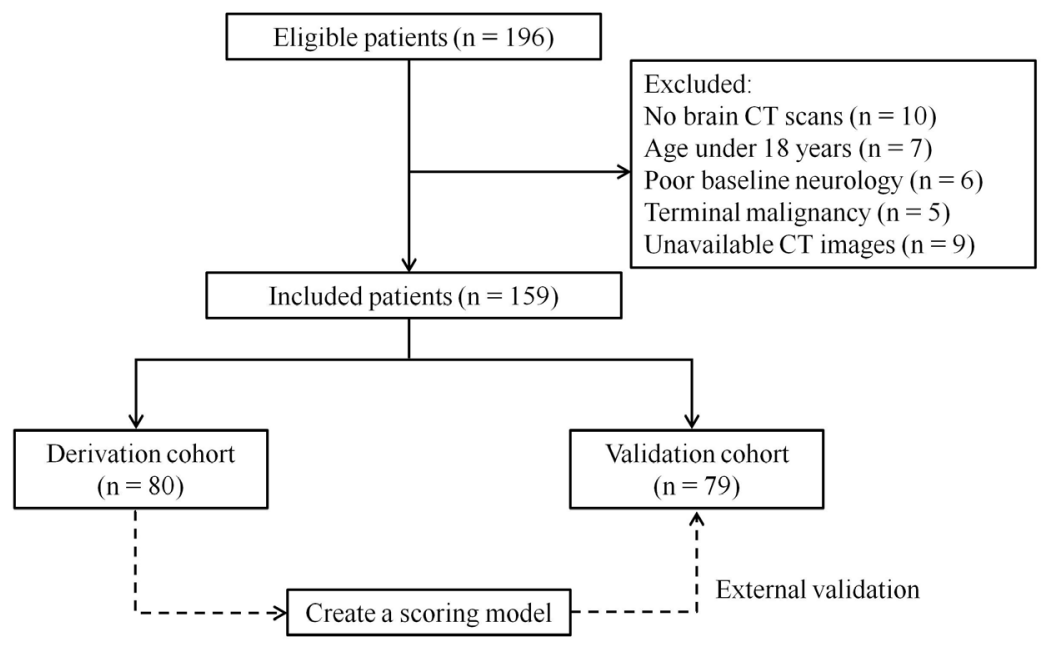

Figure 1 - Flow chart of patient enrollment. 
Table 2 - Univariate analysis of prognostic factors associated with unfavorable neurological outcome in the derivation cohort.

\begin{tabular}{|c|c|c|c|}
\hline Variables & $\begin{array}{l}\text { Unfavorable outcome } \\
\text { CPC } 3-5(n=58)\end{array}$ & $\begin{array}{l}\text { Favorable outcome } \\
\text { CPC 1-2 }(n=22)\end{array}$ & $P$-value \\
\hline Age (years), mean \pm SD & $49.35 \pm 19.53$ & $52.55 \pm 17.29$ & 0.502 \\
\hline Male, n (\%) & $40(69.0)$ & $14(63.6)$ & 0.650 \\
\hline Witnessed, n (\%) & $39(67.2)$ & $21(95.5)$ & 0.009 \\
\hline Bystander CPR, n (\%) & $36(62.1)$ & $19(86.4)$ & 0.036 \\
\hline Out-of-hospital CA, n (\%) & $35(60.3)$ & $12(54.5)$ & 0.638 \\
\hline \multicolumn{4}{|l|}{ Comorbidities, $n(\%)$} \\
\hline Coronary artery disease & $9(15.5)$ & $0(0)$ & 0.118 \\
\hline Hypertension & $22(37.9)$ & $9(40.9)$ & 0.807 \\
\hline Diabetes & $8(13.8)$ & $6(27.3)$ & 0.277 \\
\hline Stroke & $6(10.3)$ & $3(13.6)$ & 0.984 \\
\hline Initial rhythm, shockable, n (\%) & $16(27.6)$ & $13(59.1)$ & 0.009 \\
\hline Cardiac etiology, n (\%) & $27(46.6)$ & $14(63.6)$ & 0.172 \\
\hline Duration of resuscitation (min), median (IQR) & $40.00(25.75-79.75)$ & $29.50(16.75-51.75)$ & 0.035 \\
\hline Length of stay in hospital (d), median (IQR) & $11.50(5.00-23.25)$ & $18.50(14.00-24.25)$ & 0.097 \\
\hline GCS-M $\leq 2, \mathrm{n}(\%)$ & $54(93.1)$ & $7(31.8)$ & $<0.001$ \\
\hline Pupillary/corneal reflex, n (\%) & $30(51.7)$ & $22(100)$ & $<0.001$ \\
\hline \multicolumn{4}{|l|}{$G W R$, median $(I Q R)$} \\
\hline Basal ganglia & $1.15(1.08-1.24)$ & $1.27(1.22-1.37)$ & $<0.001$ \\
\hline Average & $1.20(1.09-1.31)$ & $1.32(1.24-1.44)$ & 0.001 \\
\hline NSE (ng/ml), median (IQR) & $151.60(67.23-347.35)$ & $37.00(30.32-49.95)$ & $<0.001$ \\
\hline
\end{tabular}

Table 3 - Categorical classification of each variable.

\begin{tabular}{lccc}
\hline Scores & $\mathbf{0}$ & $\mathbf{1}$ & $\mathbf{2}$ \\
\hline Witnessed/Bystander CPR & Witnessed, Bystander CPR & Witnessed, No bystander CPR & No witnessed \\
Initial rhythm & Shockable & Nonshockable & \\
Duration of resuscitation & $<30 \mathrm{~min}$ & $\geq 30 \mathrm{~min}$ & \\
GCS-M & $>2$ & $\leq 2$ & \\
Pupillary/corneal reflex & Yes & No & \\
GWR-basal ganglia & $\geq 1.20$ & $1.15-1.19$ & $\geq 1.15$ \\
NSE (ng/ml) & $<80$ & $80-159$ & $\geq 160$ \\
\hline
\end{tabular}

CPR-cardiopulmonary resuscitation; GCS-M- Glasgow Coma Scale motor score; GWR- gray-white matter ratio; NSE- neuron-specific enolase

information and the ROI location determined by the other investigator.

The NSE serum concentrations were determined in a routine clinical setting 3 days after ROSC by Roche Electro-Chemi-Luminescent-Immuno Assay (ECLIA) test kits (Roche Diagnostics, Rotkreuz, Switzerland) complying with the manufacturer's protocol. The highest NSE values obtained over $72 \mathrm{~h}$ were used in further analysis.

Outcome measurement. In accordance with the Cerebral Performance Category (CPC) ${ }^{13}$ neurological outcomes were evaluated at 30 days after CA considering both the electronic medical records of the hospitals that admitted the patients at 30 days and communication with the related family members. The CPC scale is scored as follows: 1, good recovery; 2, moderate disability; 3, severe disability; 4, comatose or vegetative state and 5, death. The patients were sorted into a favorable outcome (CPC 1-2) group or an unfavorable outcome (CPC 3-5) group.

Statistical analysis. Continuous variables are described as means \pm standard deviations or medians 
Table 4 - ROC-analysis for prediction of unfavorable neurological outcome in both derivation and validation cohorts.

\begin{tabular}{|c|c|c|c|c|c|c|c|}
\hline Prognostic factors & Cut-off value & Sensitivity (\%) & Specificity (\%) & PPV (\%) & NPV (\%) & AUC $(95 \% \mathrm{CI})$ & P-value \\
\hline \multicolumn{8}{|l|}{ Derivation cohort } \\
\hline \multicolumn{8}{|l|}{$C A N P$} \\
\hline & $\geq 3$ & 94.8 & 77.3 & 91.7 & 85.0 & \multirow{2}{*}{$0.948(0.902-0.993)$} & \multirow{2}{*}{$<0.001$} \\
\hline & $\geq 5$ & 65.5 & 100 & 100 & 52.4 & & \\
\hline GWR & $\leq 1.18$ & 65.5 & 86.4 & 92.7 & 48.7 & $0.784(0.686-0.883)$ & $<0.001$ \\
\hline \multicolumn{8}{|l|}{ Validation cohort } \\
\hline CANP & $\geq 5$ & 68.8 & 100 & 100 & 80.0 & $0.964(0.928-0.999)$ & $<0.001$ \\
\hline GWR & $\leq 1.18$ & 50.0 & 87.8 & 79.2 & 65.5 & $0.783(0.681-0.885)$ & $<0.001$ \\
\hline
\end{tabular}

PPV- positive predictive value, NPV- negative predictive value, AUC - area under the curve, CI - confidence interval, CANP -

Cardiac Arrest Neurological Prognosis, GWR - gray-white matter ratio

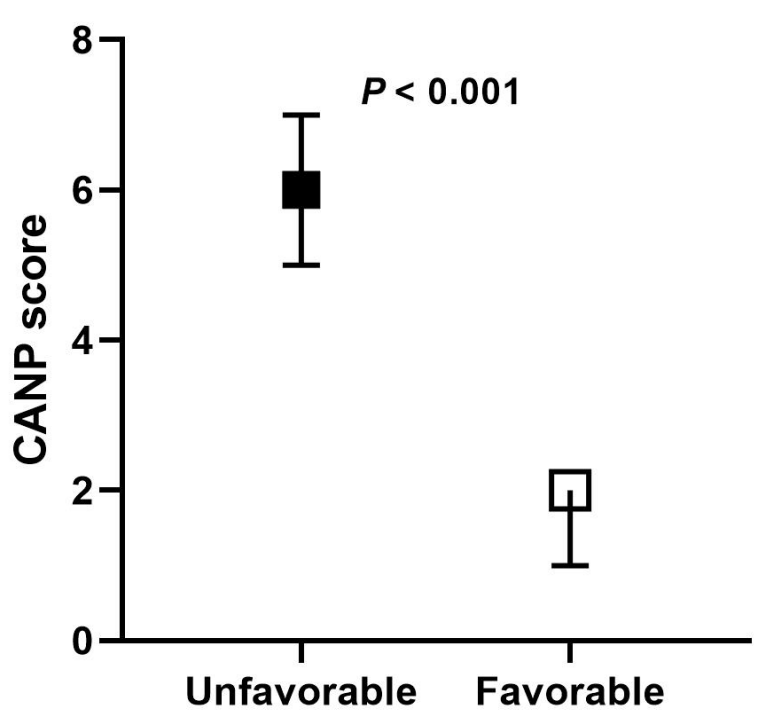

Figure 2 - Comparison of CANP scores between patients with unfavorable and favorable neurological outcome in the validation cohort. CANP- Cardiac Arrest Neurological Prognosis.

with interquartile ranges decided by their normality, and categorical variables are described as counts (percentages). The comparisons of continuous variables were performed with $\mathrm{t}$ tests or Mann-Whitney $\mathrm{U}$ tests. Categorical variables were compared by $\chi 2$ tests. For the determination of the scoring model's performance in the prediction of prognosis, discrimination and calibration were evaluated in derivation and validation cohorts respectively through receiver-operating characteristic (ROC) curve analyses. The predictive power of these prognostic factors was determined by adopting the area under the curve values and their $95 \%$ confidence intervals. Data were processed utilizing SPSS software package version 23.0 (SPSS Inc. Chicago, USA). All statistical tests were two-tailed. A $p$-value $\leq 0.05$ was of statistical significance.

Results. Clinical characteristics. The included patients' baseline clinical characteristics were summarized in Table 1. Comparison of the baseline data of the derivation cohort with those of the validation cohort revealed no differences in the distribution of age, gender and OHCA. However, the validation cohort had significantly more witnesses, less bystander CPR and resuscitation duration, as well as fewer patients with cardiac etiology. The association between prognostic factors and neurological outcome in the derivation cohort was demonstrated in Table 2. According to the CPC score obtained at 30 days after CA, 58 patients $(72.5 \%)$ were allocated to the unfavorable outcome group, while $22(27.5 \%)$ were allocated to the favorable group. Among the most pre-CA baseline variables (age, gender, and comorbidities) and CA characteristics (CA setting and cardiac etiology), no significant differences were observed between the unfavorable and favorable outcome groups $(p>0.05)$, while unwitnessed CA, no bystander CPR, initial unshockable rhythm, and longer time from CPR to ROSC were related to unfavorable outcomes $(p<0.05)$. Furthermore, significant differences were found in GWR and NSE between the 2 groups $(p<0.001)$. The absence of brainstem (pupillary/corneal) reflexes and GCS-M $\leq 2$ were also significantly correlated with unfavorable outcomes $(p<0.001)$.

A simple risk scoring model for an unfavorable outcome. Of the considered variables, we chose 8 prognostic factors (witnessed status, bystander CPR, initial rhythm, duration of resuscitation, GCS-M score, pupillary/corneal reflex, GWR-basal ganglia and NSE) that showed significant associations with the patient 
outcomes to create the Cardiac Arrest Neurological Prognosis (CANP) score. When developing the scoring model by using the variables, we first changed the continuous variables into categorical variables so that higher scores suggested poorer outcomes, and the sum of points ranged from 0 to 10 (Table 3). Next, we validated the scoring model in a separate cohort.

Prognostic performance of CANP score. The scoring system was assessed by external and internal validation in the terms of its predictive accuracy. The model performance for predicting unfavorable outcomes in both the derivation and validation cohorts was shown in Table 4. The unfavorable neurological outcome group had significantly higher CANP scores in the validation cohort $(p<0.001)$ (Figure 2). A CANP score $\geq 5$ was associated with unfavorable neurological outcomes (sensitivity 68.8\%, specificity 100\%).

Discussion. Neurological outcomes of patients resuscitated from CA are associated with various individual-related factors, such as age, comorbidities, and CA event circumstances, including unwitnessed arrest, cardiac rhythm and time to ROSC at the initial assessment. ${ }^{14}$ These factors together with clinical investigations and laboratory/imaging tests at hospital admission might already provide information about an individual's fate in terms of unfavorable neurological outcomes and mortality. ${ }^{15}$ Previous studies have focused on early neurological outcome indicators of comatose post-CA survivors. However, none of these indicators can by itself satisfactorily distinguish patients with favorable outcomes from individuals with unfavorable ones, showing that it might be necessary to establish a "proper scale" on the basis of combined predictive factors. ${ }^{16,17}$

In the CA literature, there are a large number of singleand multicenter reports in which so-called independent factors have been identified as related to different outcomes. ${ }^{4,18-19}$ More rigorous studies have identified factors by multivariate analyses that use sophisticated equations and scoring systems able to predict prognosis into consideration. As the first developed model, the score of out-of-hospital CA (OHCA) was established based on a study of 130 consecutive OHCA survivors. It gained validation in the other prospective cohort of 210 participants. The model accuracy in prediction was validated in 4 individual ICUs with 7 early variables during 2 different periods. ${ }^{5}$ Subsequently, the scores of $\mathrm{CAHP}^{6}$ namely cardiac arrest hospital prognosis and $\mathrm{CAST}^{7}$ namely the post-cardiac arrest syndrome for induced therapeutic hypothermia were obtained due to efforts to develop a predictive scoring model on the basis of data collected at hospital admission. Nevertheless, these scores failed to be validated for the clinical application. In this study, we created the CANP score based on variables associated with CA circumstances and the early phase of hospital admission and found that it performed well in the prediction of unfavorable neurological outcomes. Several notable features of this CANP scoring model should be mentioned. The model offers a relatively comprehensive system for evaluating neurological outcomes for post-CA survivors. Considering the incompleteness and availability data of premorbid status and prehospital phase, we carried out a complete analysis of eight easy-to-obtain variables concerning arrest circumstances, clinical examination, brain imaging and biomarker indicators. Additionally, we utilized another independent external cohort to validate the predictive accuracy of the model and verified its diagnostic value. This model might represent an efficient and practical approach to early neuroprognostication in China.

In previous studies, the prognostic factors involved in the scoring models were limited mostly to clinical history items, such as the witness status and initial rhythm. Information obtained by physical examination, imaging findings, or blood examination were not involved. Our data consisted of the patients' medical history, clinical exam findings, neuroimaging, and blood biomarkers, all of which actually showed strong associations with outcomes. Moreover, studies concerning predictive scores, though of some help, can also be controversial. ${ }^{20}$ Given that these models cannot show the precise possibility of outcome for an individual patient and only provide general population-based information, predictive scores should be taken into consideration very carefully. ${ }^{21}$ Predictive scores may aid in risk evaluations and decision-making, but the results are far from absolutely accurate. ${ }^{22}$ Decision regarding therapeutic strategies should be made allowing for both scoring model results and different factors, including patient preference, discussion with family members, and social considerations. It is very important that a patient's exact neurological outcomes are predicted based on decisive examinations, such as somatosensory evoked potentials, electroencephalograms or long-term observation. ${ }^{23}$

Limitations also make their appearance in our study. First, this retrospective study design was likely subjected to selection bias and other confounders. Both patients of poor baseline neurology and those in critical conditions after ROSC who cannot undergo brain CT or should have a delayed CT scan were excluded from this study. Therefore, the included cases may bias toward better neurological outcomes. Second, data in this study were collected over a relatively long period of time from different hospitals. While a standardized protocol was applied to enroll cases and assess outcomes, the risk of 
bias of a self-fulfilling prophecy still exists in this study. Third, we did not collect some potentially significant clinical and prognostic factors, such as total epinephrine dose, arterial blood gas, lactate level, activated partial thromboplastin time (APTT), end-tidal carbon dioxide (ETCO2), and SOFA score. Further studies and stratification analysis should be performed to evaluate these indicators to provide a more comprehensive scoring system. Finally, the endpoint was the outcomes at 30 days after CA in the present study. Though other predictive scores have also been established by using the outcomes at 30 days, longer-term endpoints should be determined for more accurate prediction, such as outcomes at 90 days.

In conclusion, the CANP score was developed and validated for the predicting possible neurological outcomes among comatose post-CA survivors. The likelihood of an unfavorable outcome is strong with a high specificity with a CANP score $\geq 5$. Further studies are in need to verify its effectiveness to predict the long-term prognosis of CA survivors.

\section{Acknowledgement. We would like to thank} American Journal Experts (https://www.aje.com) for English language editing.

\section{References}

1. Elmer J, Callaway CW. The Brain after Cardiac Arrest. Semin Neurol 2017; 37: 19-24.

2. Rossetti AO, Rabinstein AA, Oddo M. Neurological prognostication of outcome in patients in coma after cardiac arrest. Lancet Neurol 2016; 15: 597-609.

3. Taccone FS, Baar I, De Deyne C, Druwe P, Leqros B, Meyfroidt $G$, et al. Neuroprognostication after adult cardiac arrest treated with targeted temperature management: task force for Belgian recommendations. Acta Neurol Belg 2017; 117: 3-15.

4. Callaway CW. Neuroprognostication postcardiac arrest: translating probabilities to individuals. Curr Opin Crit Care 2018; 24: 158-164.

5. Wang CH, Huang CH, Chang WT, Tsai MS, Yu PH, Wu YW, et al. Prognostic performance of simplified out-of-hospital cardiac arrest (OHCA) and cardiac arrest hospital prognosis (CAHP) scores in an East Asian population: A prospective cohort study. Resuscitation 2019; 137: 133-139.

6. Maupain C, Bougouin W, Lamhaut L, Deye N, Diehl JL, Geri G, et al. The CAHP (Cardiac Arrest Hospital Prognosis) score: a tool for risk stratification after out-of-hospital cardiac arrest. Eur Heart J 2016; 37: 3222-3228.

7. Nishikimi M, Matsuda N, Matsui K, Takahashi K, Ejima T, Liu $\mathrm{K}$, et al. A novel scoring system for predicting the neurologic prognosis prior to the initiation of induced hypothermia in cases of post-cardiac arrest syndrome: the CAST score. Scand J Trauma Resusc Emerg Med 2017; 25: 49.

8. Martinell L, Nielsen N, Herlitz J, Karlsson T, Horn J, Wise MP, et al. Early predictors of poor outcome after out-of-hospital cardiac arrest. Crit Care 2017; 21: 96.
9. Bascom KE, Dziodzio J, Vasaiwala S, Mooney M, Patel N, McPherson J, et al. Derivation and Validation of the CREST Model for Very Early Prediction of Circulatory Etiology Death in Patients Without ST-Segment-Elevation Myocardial Infarction After Cardiac Arrest. Circulation 2018; 137: 273-282.

10. Dumas F, Bougouin W, Cariou A. Cardiac arrest: prediction models in the early phase of hospitalization. Curr Opin Crit Care 2019; 25: 204-210.

11. Dyson K, Brown SP, May S, Smith K, Koster RW, Beesems SG, et al. International variation in survival after out-of-hospital cardiac arrest: A validation study of the Utstein template. Resuscitation 2019; 138: 168-181.

12. Wang GN, Chen XF, Lv JR, Sun NN, Xu XQ, Zhang JS. The prognostic value of gray-white matter ratio on brain computed tomography in adult comatose cardiac arrest survivors. J Chin Med Assoc 2018; 81: 599-604.

13. Grossestreuer AV, Abella BS, Sheak KR, Cinousis MJ, Perman SM, Leary $\mathrm{M}$, et al. Inter-rater reliability of post-arrest cerebral performance category (CPC) scores. Resuscitation 2016; 109: 21-24.

14. Jun GS, Kim JG, Choi HY, Kang GH, Kim W, Jang YS, et al. Prognostic factors related with outcomes in traumatic outof-hospital cardiac arrest patients without prehospital return of spontaneous circulation: a nationwide observational study. Clin Exp Emerg Med 2020; 7: 14-20.

15. Sandroni C, D'Arrigo S, Cacciola S, Hoedemaekers CWE, Kamps MJA, Oddo M, et al. Prediction of poor neurological outcome in comatose survivors of cardiac arrest: a systematic review. Intensive Care Med 2020; 46: 1803-1851.

16. Oddo M, Friberg H. Neuroprognostication after cardiac arrest in the light of targeted temperature management. Curr Opin Crit Care 2017; 23: 244-250.

17. Okada Y, Kiguchi T, Irisawa T, Yamada T, Yoshiya K, Park C, et al. Development and Validation of a Clinical Score to Predict Neurological Outcomes in Patients With Out-of-Hospital Cardiac Arrest Treated With Extracorporeal Cardiopulmonary Resuscitation. JAMA Netw Open 2020; 3: e2022920..

18. Keijzer HM, Hoedemaekers CWE, Meijer FJA, Tonino BAR, Klijn CJM, Hofmeijer J. Brain imaging in comatose survivors of cardiac arrest: Pathophysiological correlates and prognostic properties. Resuscitation 2018; 133: 124-136.

19. Hawkes MA, Rabinstein AA. Neurological Prognostication After Cardiac Arrest in the Era of Target Temperature Management. Curr Neurol Neurosci Rep 2019; 19: 10.

20. Carrick RT, Park JG, McGinnes HL, Lundquist C, Brown KD, Janes WA, et al. Clinical Predictive Models of Sudden Cardiac Arrest: A Survey of the Current Science and Analysis of Model Performances. J Am Heart Assoc 2020; 9: e017625.

21. Kim J, Park YR, Lee JH, Lee JH, Kim YH, Huh JW. Development of a Real-Time Risk Prediction Model for In-Hospital Cardiac Arrest in Critically Ill Patients Using Deep Learning: Retrospective Study. JMIR Med Inform 2020; 8: e16349.

22. Saarinen S, Salo A, Boyd J, Laukkanen-Nevala P, Silfvast C, Virkkunen I, et al. Factors determining level of hospital care and its association with outcome after resuscitation from prehospital pulseless electrical activity. Scand J Trauma Resusc Emerg Med 2018; 26: 98.

23. Du L, Zheng K, Feng L, Cao Y, Niu Z, Song Z, et al. The first national survey on practices of neurological prognostication after cardiac arrest in China, still a lot to do. Int J Clin Pract 2021; 75: e13759. 